\title{
Laboreal
}

Volume 15 N$^{\circ} 2$ | 2019

Varia

\section{Subjetividad y trabajo : entre malestar y bienestar : presentación del dossier}

Subjetividade e trabalho : entre mal-estar e bem-estar : apresentação do dossier Subjectivité et travail : entre inconfort et bien-être : présentation du dossier Subjectivity and work : between discomfort and well-being : presentation of the dossier

\section{Dominique Lhuilier y Marianne Lacomblez}

\section{OpenEdition}

\section{Journals}

Edición electrónica

URL: http://journals.openedition.org/laboreal/15350

DOI: 10.4000/laboreal. 15350

ISSN: 1646-5237

\section{Editor}

Universidade do Porto

\section{Referencia electrónica}

Dominique Lhuilier y Marianne Lacomblez, « Subjetividad y trabajo : entre malestar y bienestar presentación del dossier », Laboreal [En línea], Volume 15 №2 | 2019, Publicado el 01 diciembre 2019 consultado el 24 septiembre 2020. URL : http://journals.openedition.org/laboreal/15350 ; DOI : https://doi.org/10.4000/laboreal.15350

Este documento fue generado automáticamente el 24 septiembre 2020.

\section{(c) (†) 8}

Laboreal está licenciado com uma Licença Creative Commons - Atribuição-NãoComercial 4.0 Internacional. 


\section{Subjetividad y trabajo : entre malestar y bienestar : presentación del dossier}

Subjetividade e trabalho : entre mal-estar e bem-estar : apresentação do dossier

Subjectivité et travail : entre inconfort et bien-être : présentation du dossier

Subjectivity and work : between discomfort and well-being : presentation of the dossier

Dominique Lhuilier y Marianne Lacomblez

\section{El simposio de La Habana}

1 Fue celebrado en La Habana, del 29 al 31 de octubre de 2018, un simposio "FranciaAmérica Latina" dedicado al tema : Subjetividad y trabajo : entre el malestar y el bienestar. Construcción de un nuevo paradigma de salud en el trabajo.

2 Este evento tenía como propósito reforzar las formas de cooperación entre diversos organismos, instituciones e investigadores que, desde hace mucho tiempo, tienen trabajado en varios países para la promoción de la salud de los trabajadores, tanto con la producción de trabajos científicos como con el desarrollo de acciones de prevención en los distintos contextos laborales. Así, la organización del simposio estuvo a cargo del Instituto Nacional para la Salud de los Trabajadores (Cuba) y de varias universidades, no solo cubanas, pero también argentinas, brasileñas, chilenas y francesas. El Simposio contó, además, con la colaboración de distintos socios vinculados a este tema: SELF, AFPTO, INSAT, RIPSIT, CYTED, la red TAS, RESPTO, GESTES [ $\left.{ }^{1}\right]$.

3 Fue, de esta forma, que investigadores y especialistas provenientes de contextos socioculturales diversos (Argentina, Brasil, Chile, Colombia, Cuba, Uruguay, Francia, Portugal, Quebec y Suiza), con diferentes experiencias profesionales, pero todos comprometidos en un objetivo común de humanización del trabajo, han colaborado 
durante esos tres días : más de 450 participantes y 181 comunicaciones (41 en español, 75 en portugués y 65 en francés).

4 Este dosier, publicado por la revista Laboreal, no pretende ni puede contar el gran número de temas y debates de esas jornadas [2]. Se ha optado, así, por presentar aquí algunas aportaciones que revelan el desarrollo de la psico(socio)logia en el ámbito de la salud en el trabajo y, asimismo, de la medicina del trabajo.

5 Se reúnen, de este modo, cinco artículos que, teniendo como base las respectivas comunicaciones presentadas en el simposio, proponen análisis contextualizadas de la investigación y de las acciones llevadas a cabo en Argentina, Brasil, Chile, Colombia y Cuba.

\section{Balance de la construcción de un nuevo paradigma de salud en el trabajo}

6 La problemática elegida privilegia el análisis de las relaciones entre trabajo y salud, basado en comparaciones internacionales y estudios multidisciplinarios. Abordar la relación salud/trabajo presupone la aclaración de los conceptos de ambos los términos y de sus relaciones reciprocas. Sin embargo, si se impone aclarar los términos en el plan del conocimiento científico, en el plan de la acción el problema es igualmente relevante. Igualmente, tales cuestiones tienen que ser necesariamente repuestas en los contextos económicos, políticos, sociales y culturales de cada país.

7 Aunque la tarea sea compleja y las situaciones diversas, hay algunas líneas de reflexión comunes que emergen de los distintos intercambios que, por su turno, permiten esbozar una trama para los próximos encuentros - y que pretendemos sistematizar aquí :

8 2.1. Tanto en el sentido común como en acepciones científicas, el término trabajo es polisémico. Cuando se confunde con la tarea definida o con la profesión, la dimensión de la actividad real desaparece por completo. Por otro lado, la tónica en la actividad puede acabar por subestimar la división social del trabajo, las relaciones sociales de trabajo o las normas jurídicas previstas para su encuadramiento. Asimismo, en muchos países, aquello a que llamamos trabajo informal (fuera de cualquier relación contractual establecida) corresponde a una parte primordial de la producción y de las condiciones de vida de la población trabajadora. Esto obliga a rever las concepciones del trabajo esencialmente relacionadas con la esfera del empleo (por ejemplo, ¿cómo concebir la relación tarea/actividad en esos contextos laborales ?).

9 2.2. Las comparaciones internacionales pueden llevar a repensar la asimilación del trabajo al empleo. Estas pueden ayudar a tener en cuenta otros tipos de actividades además de las que se basan en los salarios. Hay numerosas actividades que aseguran las mismas funciones que el trabajo, entendido en el sentido común del término : función utilitaria, social e identitaria. Esta perspectiva vuelve a asociar la vida de trabajo con la vida fuera del trabajo para tener en cuenta los procesos de interacción en el ámbito de las actividades, de su regulación y desregulación. La relación laboral y las actividades desarrolladas en ella, además de caracterizar una esfera autónoma, se fusionan con toda la vida pasada y actual. Son justamente esas actividades desarrolladas en el ámbito del trabajo y fuera de él que constituyen, con el paso del tiempo, conocimientos y 
modos de saber-hacer que pueden ser transferidos de una esfera hacia otra. Esta cuestión se relaciona, sobre todo, con las cuestiones de género.

2.3. Incluso cuando se considera la actividad (enmarcada o no en la subordinación constitutiva de un contrato de trabajo), el trabajo puede también designar cosas muy diferentes, como, por ejemplo : una "actividad inconsciente", semejante a la del obrero taylorista, o una actividad laboral siempre única, aunque fundamentalmente vinculada a la actividad de los otros, especialmente a través del colectivo de trabajadores, un instrumento de la actividad individual. En realidad, la actividad y la subjetividad no son disociables, considerándose la subjetividad tanto en sus relaciones con los marcos simbólicos que orientan el sentido como en sus relaciones inevitables con la corporalidad.

11 2.4. La relación entre salud y trabajo está involucrada en controversias epistemológicas profundas y, en simultaneo, en cuestiones sociales igualmente importantes, tanto en Latinoamérica como en Canadá o Europa. Es sabido que la percepción de la relación entre salud y trabajo se construyó históricamente mediante el prisma enfermedad y trabajo, y que los efectos deletéreos del trabajo en la salud son, desde hace mucho tiempo, objeto de preocupación. Son esos primeros análisis sobre la naturaleza de la relación entre salud y trabajo que se encuentran en la base de los trabajos actuales que analizan las enfermedades propias de cada oficio, o los daños para la salud del nuevo trabajo industrial que conjuga explotación y miseria. Y también estuvieron en la base de las intervenciones de los movimientos sociales y del Estado mismo que marcaron la historia de una herencia memorable : una herencia materializada en el sistema actual, atravesada por una combinación de luchas, movilizaciones, encuestas, legislaciones e instituciones, y que tuvieron por objetivo la prevención y el resarcimiento de los daños causado por el trabajo.

12 Sin embargo, estos enfoques de la salud en el trabajo deben ser objeto de revisión a la luz de los actuales contextos sociales, históricos y culturales de países con diferentes referenciales, diferentes reglamentaciones y diferentes sistemas de evaluación de la salud de los trabajadores.

13 2.5. El esquema determinista causa-efecto, que ha dominado durante mucho tiempo el enfoque de las patologías profesionales, puede todavía prevalecer, particularmente en Cuba, donde los compañeros esperaban de este Simposio la posibilidad de insertarse en la dinámica de la construcción de un nuevo paradigma de la salud en el trabajo. Se trata, en realidad, de hacer el modelo inicial más complejo para integrar enfoques multifactoriales y diacrónicos, como, por ejemplo : tener en cuenta la asociación y la potencialización de riesgos, considerar el período de latencia entre la exposición a los riesgos y las manifestaciones clínicas, reconstituir las trayectorias de exposición a situaciones nocivas. Se reevaluará, de este modo, la hipótesis patogénica, inspirada en el modelo de la toxicología clásica, a la luz de un enfoque abierto a la complejidad de las historias profesionales reales. Podemos, entonces, considerar una revisión de la noción de nocividad del trabajo, subrayando las desigualdades en relación al riesgo, dado que las condiciones socio-económicas que caracterizan la vida de las/los que trabajan (estado nutricional, educación, posibilidad de acceso a la información, calidad concreta/real de las políticas públicas, alojamiento, ...) pueden decuplicar los efectos de la exposición a "factores de riesgo" nocivos. Es, por consiguiente, por razones científicas y no solo éticas o políticas, que se impone un enfoque "integrado", multiprofesional e interdisciplinario. 
14 2.6. Por otra parte, aunque la problematización del modelo toxicológico inicial trae, también, para primer plano los trabajadores que se quieren mantener sujetos de su trabajo y actores de sus condiciones de trabajo. Se abre, así, un campo de investigación y de intervención que no deja en la sombra el papel determinante del trabajo como agente de salud, es decir, en el desarrollo y en la construcción de la salud. Un campo todavía más extenso cuando se aborda la manutención en actividad de aquellos que, en un número cada vez mayor, viven con una salud frágil, o asimismo aquellos que viven "sin trabajo" al margen del mercado de empleo. En este dominio, la producción científica es aun claramente insuficiente, pese a que la psicopatología del trabajo haya legado una tradición considerable en lo que toca a las funciones psicológicas y sociales del trabajo en el restablecimiento y desarrollo de la salud psíquica.

2.7. La renovación de los enfoques se impone también en virtud de los efectos de las transformaciones del trabajo en los contextos sociales, económicos y políticos de cada uno de los distintos países. La globalización económica vino, en realidad, cuestionar las condiciones del ejercicio del poder soberano del Estado en coyunturas nacionales políticamente diferentes. De hecho, el comercio internacional, la libre circulación de capitales y, al mismo tiempo, el desarrollo de los mercados financieros internacionales, tienden a interferir y a influenciar el ejercicio del poder de los Estados. Pues bien, la liberalización mundial de los factores de producción, tales como el trabajo y el capital, no se procesa de la misma manera ni con la misma intensidad. De este modo, si por un lado los flujos migratorios son, hoy en día, estrictamente controlados en muchos países, la libertad de circulación de capitales, por otro lado, es intensamente fomentada a escala mundial, a pesar de que, en algunos países, como Cuba, sigue todavía, y por diversas razones, siendo objeto de prácticas restrictivas.

\section{La continuación de los debates}

Este evento científico ha permitido reunir a un gran número de investigadores y profesionales relacionados con la problemática de la salud en el trabajo, comprometidos con el intercambio de experiencias y con el/los diálogo(s) entre los varios enfoques disciplinarios (psicología del trabajo, ergonomía, sociología, ciencias de la gestión, ciencias de la educación, derecho, filosofía, etc.). Al someter a debate el/los concepto(s) de salud - entendida en sus dimensiones física, psíquica y social - y el concepto de trabajo, bien como las interacciones entre ambos, se ha pretendido hacer una aportación para una producción científica internacional que, por tener, con frecuencia, al mundo anglófono como referencia, tiende a subestimar el carácter innovador de la investigación y de las intervenciones llevadas a cabo en regiones hispanófonas y lusófonas.

17 Sin duda que el desafío continuará, desde luego porque el segundo Simposio FranciaAmérica Latina, que se celebrará en João Pessoa, en Brasil, a finales de octubre del 2020, tendrá como tema central Trabajar: ¿para hacer lo qué? (https://eitajp.wixsite.com/ jp2020). 


\section{NOTAS}

1. Société d'Ergonomie de Langue Française, Association Française de Psychologie du travail et des Organisations, Instituto Nacional de Salud de los Trabajadores, Red Iberoamericana de Promoción de la Seguridad Integral en el Trabajo, Programa Iberoamericano de Ciencia y Tecnología para el Desarrollo, Réseau de Recherche de Psychologie du Travail et des Organisations, Groupe d'Etudes Sur le Travail et la Souffrance au travail.

2. Se está finalizando una obra colectiva, en tres idiomas, compuesta por 24 textos resultantes de las comunicaciones presentadas durante el Simposio. Será publicada en 2020 por la editorial L'Harmattan, en versión electrónica y en papel, bajo el título: Subjectivité et travail. Construction d'un nouveau paradigme de la santé au travail [Subjetividad y trabajo. Construcción de un nuevo paradigma de salud en el trabajo].

\section{AUTORES}

\section{DOMINIQUE LHUILIER}

Équipe Psychosociologie du travail et de la formation, Centre de recherche sur le travail et le développement, Conservatoire National des Arts et Métiers [Grupo de Psicologia do Trabalho e da Formação, Centro de Investigação do Trabalho e do Desenvolvimento, Conservatório Nacional das Artes e Ofícios]. 41 rue Gay Lussac, 75005 Paris, France.

dominique.lhuilier@lecnam.net

\section{MARIANNE LACOMBLEZ}

Centro de Psicologia da Universidade do Porto, Faculdade de Psicologia e Ciências da Educação, Universidade do Porto. Rua Alfredo Allen, 4200-135, Porto, Portugal.

lacomb@fpce.up.pt 